\title{
REGULARITY CRITERIA FOR INTEGRAL AND MEROMORPHIC FUNCTIONS
}

\author{
BY \\ J. M. ANDERSON(1)
}

1. Introduction. In this paper we shall consider functions $f(z)$ which are meromorphic in the plane (hereinafter called meromorphic). In particular we consider integral functions. Throughout the paper we shall assume familiarity with the standard notation of the Nevanlinna theory,

$$
T(r)=T(r, f), N(r, a), m(r, a), \delta(a, f) \cdots
$$

and with the first fundamental theorem (see e.g. [7]). We define

$$
\begin{array}{ll}
M(r)=M(r, f)=\max |f(z)| & (|z|=r), \\
\mu(r)=\mu(r, f)=\min |f(z)| & (|z|=r),
\end{array}
$$

using $\mu(r)$ instead of $m(r)$ for the minimum modulus to avoid confusion with the schmiegungsfunktion $m(r, f)$. We shall assume that $f(z)$ is transcendental i.e. that

$$
\log r=o(T(r)) \quad(r \rightarrow \infty)
$$

and also that $f(0)=1$. It is easily seen in the sequel that this involves no loss of generality.

If $f(z)$ is an integral function then for $r$ sufficiently large [7, p. 18]

$$
T(r, f) \leqq \log M(r, f) \leqq \frac{R+r}{R-r} T(R, f) \quad(0<r<R) .
$$

From this it is easily deduced that the order or type of $f(z)$ is the same whether it is defined by $T(r, f)$ or $\log M(r, f)$. We note in particular that

$$
\begin{aligned}
& \liminf _{r \rightarrow \infty} \frac{T(r, f)}{r^{\rho}}>0 \Leftrightarrow \liminf _{r \rightarrow \infty} \frac{\log M(r, f)}{r^{\rho}}>0, \\
& \liminf _{r \rightarrow \infty} \frac{T(r, f)}{r^{\rho}}<\infty \Leftrightarrow \liminf _{r \rightarrow \infty} \frac{\log M(r, f)}{r^{\rho}}<\infty .
\end{aligned}
$$

If $f(z)$ is an integral function of order $\rho<1$ then [13], [14]

Received by the editors April 26, 1965.

(1) The author acknowledges receipt of support from the U.S. Air Force Office of Scientific Research (Contract No. AF-AFOSR-393-63) during the preparation of this paper. 


$$
\underset{r \rightarrow \infty}{\lim \sup } \frac{N(r, 0)}{\log M(r)} \geqq \frac{\sin \pi \rho}{\pi \rho} .
$$

Since $T(r, f) \geqq N(r, 0)+O(1)$ we conclude

$$
\lim _{r \rightarrow \infty} \frac{T(r)}{\log M(r)} \geqq \frac{\sin \pi \rho}{\pi \rho} .
$$

Consider the integral function $F(z)$ with real negative zeros for which $(0<\rho<1)$

$$
n(r, 0) \sim A r^{\rho} \quad(r \rightarrow \infty) .
$$

Then, as is well known (see e.g. [2])

$$
\begin{aligned}
N(r, 0) & \sim \frac{A}{\rho} r^{\rho} \quad(r \rightarrow \infty), \\
\log M(r, F) & =\log F(r) \sim \frac{A \pi}{\sin \pi \rho} r^{\rho} \quad(r \rightarrow \infty), \\
T(r, F) & =m(r, F) \sim \frac{1}{2 \pi} \frac{A \pi}{\sin \pi \rho} r^{\rho} \quad \int_{-\pi}^{\pi}(\cos \rho \theta)^{+} d \theta, \\
& =\frac{A}{\rho} r^{\rho} \quad(r \rightarrow \infty) \quad\left(0<\rho \leqq \frac{1}{2}\right) \\
& =\frac{A}{\rho \sin \pi \rho} r^{\rho} \quad(r \rightarrow \infty) \quad\left(\frac{1}{2}<\rho<1\right) .
\end{aligned}
$$

Thus (1.4) is best possible for $0<\rho<1$, and (1.5) is best possible for $0<\rho \leqq \frac{1}{2}$.

2. The classical Wiman-Heins theory [8] and its extensions by Kjellberg [9], [10] lead one to expect that the integral functions which only just attain the growth demanded by (1.4) and (1.5) would have regular growth. We have the following theorems.

THEOREM 1. Let $f(z)$ be an integral function with $f(0)=1$ such that for some $\rho$, $0<\rho \leqq \frac{1}{2}$,

$$
\pi \rho T(r) \leqq \sin \pi \rho \log M(r)
$$

for all $r>0$. Then

$$
\beta=\liminf _{r \rightarrow \infty} \frac{T(r)}{r^{\rho}}>0
$$

If, further, $\beta<\infty$, then

$$
T(r) \sim \beta r^{\rho} \quad(r \rightarrow \infty) .
$$

THEOREM 2. Let $f(z)$ be an integral function with $f(0)=1$ and such that for 
some $\rho, 0<\rho<1$

$$
\pi \rho N(r, 0) \leqq \sin \pi \rho \log M(r)
$$

for all $r>0$. Then

$$
\beta^{\prime}=\lim _{r \rightarrow \infty} \inf \frac{\log M(r)}{r^{\rho}}>0 .
$$

If, further, $\beta^{\prime}<\infty$, then

$$
\begin{aligned}
\log M(r) & \sim \beta^{\prime} r^{\rho} \quad(r \rightarrow \infty), \\
N(r, 0) & \sim \frac{\beta^{\prime} \sin \pi \rho}{\pi \rho} \quad(r \rightarrow \infty) .
\end{aligned}
$$

It is of interest to state the following corollary, which is implicit in some recent work of Edrei [3] (see also [6]).

COROLLARY 1. Let $f(z)$ be an integral function of lower order $\lambda, 0<\lambda<1$, then

$$
\limsup _{r \rightarrow \infty} \frac{T(r)}{\log M(r)} \geqq \limsup _{r \rightarrow \infty} \frac{N(r, 0)}{\log M(r)} \geqq \frac{\sin \pi \lambda}{\pi \lambda} .
$$

Proof. The first inequality is immediate. To prove the second let $\rho$ be any number greater than $\lambda$. Then

$$
\liminf _{r \rightarrow \infty} \frac{\log M(r)}{r^{\rho}}=0 .
$$

Thus by Theorem 2 there exists a sequence $\left\{r_{v}\right\}$ say, of values of $r$ tending to infinity, such that

$$
\pi \rho N\left(r_{v}, 0\right)>\sin \pi \rho \log M\left(r_{v}\right),
$$

i.e.

$$
\limsup _{r \rightarrow \infty} \frac{N(r, 0)}{\log M(r)} \geqq \frac{\sin \pi \rho}{\pi \rho} .
$$

The result follows on letting $\rho \rightarrow \lambda$.

If $\rho=1$ the condition (2.1) implies that $f(z)$ has 0 as a Picard (and a fortiori as a Borel) exceptional value. For such functions

$$
\log M(r) \sim \alpha r^{n} \quad(r \rightarrow \infty)
$$

for some $\alpha>0$ and positive integer $n$. Thus

$$
\beta=\liminf _{r \rightarrow \infty} \frac{\log M(r)}{r}>0
$$

and if $\beta<\infty$ then 


$$
\log M(r) \sim \beta r \quad(r \rightarrow \infty) .
$$

Theorem 1 can thus be considered as an extension of this result to fractional orders.

Theorem 1 is easily deduced from Theorem 2 as follows: Suppose that for $r \geqq r_{0}$

$$
\pi \rho T(r) \leqq \sin \pi \rho \log M(r) .
$$

Then, by the first fundamental theorem, since $f(0)=1$

$$
\pi \rho N(r, 0) \leqq \pi \rho T(r) \leqq \sin \pi \rho \log M(r) .
$$

Thus by Theorem 2

$$
\beta^{\prime}=\liminf _{r \rightarrow \infty} \frac{\log M(r)}{r^{\rho}}>0
$$

and so by (1.2)

$$
\beta=\liminf _{r \rightarrow \infty} \frac{T(r)}{r^{\rho}}>0 .
$$

Also if $\beta<\infty$ then by (1.3) $\beta^{\prime}<\infty$. Hence by Theorem 2

$$
\begin{array}{rlrl}
\sin \pi \rho \log M(r) & \sim \beta^{\prime} \sin \pi \rho r^{\rho} & & (r \rightarrow \infty), \\
\pi \rho N(r, 0) & \sim \beta^{\prime} \sin \pi \rho r^{\rho} & (r \rightarrow \infty)
\end{array}
$$

and thus by the inequality (2.2)

$$
T(r) \sim \frac{\beta^{\prime} \sin \pi \rho}{\pi \rho} \quad(r \rightarrow \infty)
$$

as required.

3. For the case $\rho>\frac{1}{2}$ it is a conjecture of Paley [12] that for an integral function of order $\rho$

$$
\limsup _{r \rightarrow \infty} \frac{T(r)}{\log M(r)} \geqq \frac{1}{\pi \rho} .
$$

The example of $\$ 1$ shows that the result would be sharp for $\frac{1}{2}<\rho<1$. The MittagLeffler functions [7, p. 19] show that it would be sharp for $\rho>1$. This conjecture is unproved, though Gol'dberg, [5], has shown that it is true with the additional assumption that there exists a $\theta$ for which

$$
\log \left|f\left(r e^{i \theta}\right)\right| \sim \log M(r) \quad(r \rightarrow \infty) .
$$

It is clear from our proof of Theorem 1 that it remains true for $\frac{1}{2}<\rho<1$. 
If the above conjecture of Paley is correct, however, the theorem would be vacuously true. Unfortunately the present results shed no light on the conjecture.

4. Theorem 1 can be extended, at any rate in part, to meromorphic functions. We have

THEOREM 3. Let $f(z)$ be meromorphic with $f(0)=1$ and such that for some $\rho, 0<\rho<\frac{1}{2}$,

$$
\pi \rho T(r) \leqq \sin \pi \rho \log M(r)+\pi \rho \cos \pi \rho N(r, \infty)
$$

for all $r>0$. Then

$$
\beta=\liminf _{r \rightarrow \infty} \frac{T(r)}{r^{\rho}}>0
$$

If, further, $\beta<\infty$ then

$$
\alpha=\limsup _{r \rightarrow \infty} \frac{T(r)}{r^{\rho}}<\infty .
$$

Theorem 3 is an immediate corollary of the following theorem.

THEOREM 4. Let $f(z)$ be meromorphic in the plane and such that for some $\rho, 0<\rho<1$, either

$$
\pi \rho N(r, 0) \leqq \sin \pi \rho \log M(r)+\pi \rho \cos \pi \rho N(r, \infty)
$$

or

$$
\sin \pi \rho \log \mu(r) \leqq \pi \rho \cos \pi \rho N(r, 0)-\pi \rho N(r, \infty)
$$

for all $r>0$. Then

$$
\beta=\lim _{r \rightarrow \infty} \inf \frac{T(r)}{r^{\rho}}>0
$$

If, further, $\beta<\infty$ then

$$
\alpha=\limsup _{r \rightarrow \infty} \frac{T(r)}{r^{\rho}}<\infty .
$$

Remarks 1. Condition (4.2) is just condition (4.1) applied to $F(z)=(f(z))^{-1}$, and so it suffices just to consider (4.1).

2. The inequality (4.2) and its conclusion have been used by Ostrovskii [11] to show that for a meromorphic function of lower order $\lambda<\frac{1}{2}$,

$$
\underset{r \rightarrow \infty}{\lim \sup } \frac{\log ^{+} \mu(r, f)}{T(r)} \geqq \pi \lambda(\operatorname{cosec} \pi \lambda)(\cos \pi \lambda-1+\delta(\infty)) .
$$

The result is sharp. An example to show this is easily constructed with the method of $[7$, p. 117]. 
3. It is an open question whether under the hypotheses of Theorems 3 and 4 we can conclude that $\alpha=\beta$ i.e. that $f(z)$ has perfectly regular growth in the sense of Valiron.

In the proof of Theorem 4 we can prove the following slightly more general theorem:

THEOREM 5. Let $f(z)$ be meromorphic and such that, for some $\rho, 0<\rho<1$, given $\varepsilon>0$

$$
\int_{r_{1}}^{r_{2}}(\pi \rho N(r, 0)-\sin \pi \rho \log M(r)-\pi \rho \cos \pi \rho N(r, \infty)) \frac{d r}{r^{1+\rho}}<\varepsilon,
$$

for all $r_{2}>r_{1}>r(\varepsilon)$ or

$$
\pi \rho N(r, 0) \leqq \sin \pi \rho \log M(r)+\pi \rho \cos \pi \rho N(r, \infty)+O(\log r) \quad(r \rightarrow \infty) .
$$

If $\beta<\infty$ then $\alpha<\infty$ and if (4.4) holds $\beta>0$.

5. The proof of Theorem 4 uses results similar to those in [6]. We also use the techniques developed by Kjellberg.

LEMMA 1. Let

$$
F(z)=\frac{F_{1}(z)}{F_{2}(z)}=\prod_{n=1}^{\infty}\left(1+\frac{z}{a_{n}}\right) / \prod_{m=1}^{\infty}\left(1-\frac{z}{b_{m}}\right), \quad a_{n}>0, b_{m}>0,
$$

be meromorphic and of order less than one. Then there exist constants $K, k$, depending only on $F(z)$ satisfying $0<k<K<\infty$, such that for any $r_{2}>r_{1}>0$, $0<\rho<1$,

$$
\begin{aligned}
\int_{r_{1}}^{r_{2}}(\pi \rho N(r, 0)- & \sin \pi \rho \log |F(r)|-\pi \rho \cos \pi \rho N(r, \infty)) \frac{d r}{r^{1+\rho}} \\
> & k \frac{T\left(r_{1}, F\right)}{r_{1}^{\rho}}-K \frac{T\left(2 r_{2}, F\right)}{r_{2}^{\rho}} .
\end{aligned}
$$

Proof. Let $C$ be the contour consisting of the line segments $r_{1} \leqq t \leqq r_{2}$, $-r_{2} \leqq t \leqq-r_{1}$ and the semicircles $|z|=r_{1}, 0<\arg z<\pi$, and $|z|=r_{2}$, $0<\arg z<\pi$, with indentations, of radius $\delta$ say, around the zeros and poles of $F(z)$. Consider

$$
\int_{c} \frac{\log F(z)}{z^{1+\rho}} d z
$$

We consider that branch of $z^{1+\rho}$ which is real for $z>0$ and that branch of $\log F(z)$ for which $\log F_{1}(z)$ is real for $z>0$ and $\log F_{2}(z)$ real for $z<0$. Since $F(z)$ is meromorphic $\log F(z)$ has only logarithmic singularities at the zeros and poles 
of $F(z)$. The contribution to the integrand along any indentation is therefore $O(\delta \log 1 / \delta)$ as $\delta \rightarrow 0$. Since $F(z)$ is analytic inside the contour we obtain on letting $\delta \rightarrow 0$,

$$
\begin{aligned}
0=\int_{r_{1}}^{r_{2}} & \left(\log F(r)-e^{-i \pi \rho} \log F(-r)\right) \frac{d r}{r^{1+\rho}} \\
& +\frac{i}{r_{2}^{\rho}} \int_{0}^{\pi} e^{-i \rho \theta} \log F\left(r_{2} e^{i \theta}\right) d \theta-\frac{i}{r_{1}^{\rho}} \int_{0}^{\pi} e^{-i \rho \theta} \log F\left(r_{1} e^{i \theta}\right) d \theta .
\end{aligned}
$$

On multiplying through by $e^{i \pi \rho}$ and taking real parts we obtain,

$$
\begin{gathered}
\int_{r_{1}}^{r_{2}}(\pi n(r, 0)-\sin \pi \rho \log |F(r)|-\pi \cos \pi \rho n(r, \infty)) \frac{d r}{r^{1+\rho}} \\
=r_{1}^{-\rho} P\left(r_{1}\right)-r_{2}^{-\rho} P\left(r_{2}\right)
\end{gathered}
$$

where

$$
P(r)=-\int_{0}^{\pi}\left(\cos \rho(\pi-\theta) \log \left|F\left(r e^{i \theta}\right)\right|-\sin \rho(\pi-\theta) \arg F\left(r e^{i \theta}\right)\right) d \theta .
$$

Now

$$
\int_{r_{1}}^{r_{2}} \frac{n(r, 0) d r}{r^{1+\rho}}=r_{2}^{-\rho} N\left(r_{2}, 0\right)-{\overline{r_{1}}}^{-\rho} N\left(r_{1}, 0\right)+\rho \int_{r_{1}}^{r_{2}} \frac{N(r, 0) d r}{r^{1+\rho}} .
$$

Thus we obtain

$$
\begin{gathered}
\int_{r_{1}}^{r_{2}}(\pi \rho N(r, 0)-\sin \pi \rho \log |F(r)|-\pi \rho \cos \pi \rho N(r, \infty)) \frac{d r}{r^{1+\rho}} \\
=r_{1}^{-\rho} Q\left(r_{1}\right)-r_{2}^{-\rho} Q\left(r_{2}\right)
\end{gathered}
$$

where

$$
Q(r)=\pi N(r, 0)-\pi \cos \pi \rho N(r, \infty)+P(r) .
$$

An application of Jensen's theorem [7, formula (1.5)] yields

$$
\begin{aligned}
Q(r)= & \pi(1-\cos \pi \rho) N(r, \infty) \\
& +\int_{0}^{\pi}(1-\cos \rho(\pi-\theta)) \log \left|F\left(r e^{i \theta}\right)\right| \\
& +\sin \rho(\pi-\theta) \arg F\left(r e^{i \theta}\right) d \theta,
\end{aligned}
$$

since $F(z)$ is symmetric with respect to the real axis. Now

$$
\pi(1-\cos \pi \rho) N(r, \infty)<2 \pi T(r)
$$




$$
\begin{aligned}
\int_{0}^{\pi}\left(1-\cos \rho(\pi-\theta) \log \left|F\left(r e^{i \theta}\right)\right|\right. & <2 \pi T(r) \\
\int_{0}^{\pi} \sin \rho(\pi-\theta) \arg F\left(r e^{i \theta}\right) d \theta & <[\pi n(r, 0)+\pi n(r, \infty)] \int_{0}^{\pi} \sin \rho(\pi-\theta) d \theta \\
& <\pi^{2}[n(r, 0)+n(r, \infty)] .
\end{aligned}
$$

Now

$$
n(r, 0) \log 2=n(r, 0) \int_{r}^{2 r} \frac{d t}{t}<\int_{r}^{2 r} \frac{n(t, 0) d t}{t}<N(2 r, 0)<T(2 r) .
$$

Similarly for $n(r, \infty)$, and so we obtain

$$
Q(r)<4 \pi T(r)+\frac{2 \pi^{2}}{\log 2} T(2 r)<K T(2 r)
$$

The left-hand inequality is not so immediate but it follows from the fact that $\psi(\theta)=1-\cos \rho(\pi-\theta)$ is a decreasing function of $\theta$ for $0<\theta<\pi$ and that

$$
\begin{aligned}
m(r, F) & =\frac{1}{\pi} \int_{0}^{\gamma} \log \left|F\left(r e^{i \theta}\right)\right| d \theta, \\
m\left(r, \frac{1}{F}\right) & =\frac{1}{\pi} \int_{\gamma}^{\pi} \log \left|F\left(r e^{i \theta}\right)\right| d \theta
\end{aligned}
$$

for some $\gamma=\gamma(r)$ satisfying $0 \leqq \gamma \leqq \pi$.

We prove the inequality only in the case $0 \leqq \gamma \leqq \pi / 3$, the cases when $\pi / 3<\gamma \leqq 2 \pi / 3$ and $2 \pi / 3<\gamma \leqq \pi$ being similar. Since

$$
\sin \rho(\pi-\theta) \arg F\left(r e^{i \theta}\right) \geqq 0
$$

for $0 \leqq \theta \leqq \pi$ we have

$$
\begin{aligned}
Q(r) \geqq & \pi(1-\cos \pi \rho) N(r, \infty)+\int_{0}^{\pi}(1-\cos \rho(\pi-\theta)) \log \left|F\left(r e^{i \theta}\right)\right| d \theta \\
\geqq & \pi(1-\cos \pi \rho) N(r, \infty)+\pi(1-\cos \rho(\pi-\gamma)) m(r, \infty) \\
& +\int_{\gamma}^{\pi}(1-\cos \rho(\pi-\theta)) \log \left|F\left(r e^{i \theta}\right)\right| d \theta .
\end{aligned}
$$

Now $\log \left|F\left(r e^{i \theta}\right)\right|$ is a decreasing function of $\theta$ and is less than zero for $\gamma<\theta \leqq \pi$. Thus

$$
\int_{2 \pi / 3}^{\pi} \log \left|F\left(r e^{i \theta}\right)\right| d \theta<\frac{1}{3} \int_{\gamma}^{\pi} \log \left|F\left(r e^{i \theta}\right)\right| d \theta=-\frac{\pi}{3} m(r, 0) .
$$

\section{Hence,}




$$
\begin{aligned}
Q(r) \geqq & \pi(1-\cos \rho(\pi-\gamma)) T(r, F) \\
& +\left\{\int_{\gamma}^{2 \pi / 3}+\int_{2 \pi / 3}^{\pi}\right\}(1-\cos \rho(\pi-\theta)) \log \left|\left(F r e^{i \theta}\right)\right| d \theta \\
\geqq & \pi(1-\cos \rho(\pi-\gamma)) T(r, F)+(1-\cos \rho(\pi-\gamma)) \int_{\gamma}^{2 \pi / 3} \log \left|F\left(r e^{i \theta}\right)\right| d \theta \\
& +\left(1-\cos \frac{\pi \rho}{3}\right) \int_{2 \pi / 3}^{\pi} \log \left|F\left(r e^{i \theta}\right)\right| d \theta \\
= & \pi(1-\cos \rho(\pi-\gamma)) T(r, F)-\pi(1-\cos \rho(\pi-\gamma)) m(r, 0) \\
& +\left(\operatorname{cosp} \rho(\pi-\gamma)-\cos \frac{\pi \rho}{3}\right) \int_{2 \pi / 3}^{\pi} \log \left|F\left(r e^{i \theta}\right)\right| d \theta \\
> & \pi(1-\cos \rho(\pi-\gamma)) T(r, F)-\pi(1-\cos \rho(\pi-\gamma)) m(r, 0) \\
& +\frac{\pi}{3}\left(\cos \frac{\pi \rho}{3}-\cos \rho(\pi-\gamma)\right) m(r, 0)
\end{aligned}
$$

by (5.4). Therefore by the first fundamental theorem

$$
\begin{aligned}
Q(r) & \geqq \frac{\pi}{3}\left[\cos \frac{\pi \rho}{3}-\cos \rho(\pi-\gamma)\right] T(r, F) \\
& \geqq \frac{\pi}{3}\left(\cos \frac{\pi \rho}{3}-\cos \frac{2 \pi \rho}{3}\right) T(r, F)
\end{aligned}
$$

since $0 \leqq \gamma<\pi / 3$. This completes the proof of the lemma.

6. Proof of Theorem 4. If $f(z)$ has only finitely many zeros and poles then, since we are assuming that $f(z)$ is transcendental

$$
f(z)=\frac{P_{1}(z)}{P_{2}(z)} \exp \phi(z),
$$

where $P_{1}, P_{2}$ are polynomials and $\phi(z)$ is an integral function. From this we conclude that $f(z)$ has lower order at least 1 and so

$$
\beta=\lim _{r \rightarrow \infty} \inf \frac{T(r)}{r^{\rho}}=\infty
$$

for any $\rho, 0<\rho<1$, and so the theorem is proved.

Now, following Kjellberg we choose $R$ sufficiently large so that $f(z)$ has $N$ zeros and $M$ poles in $|z|<R$ where $\max (M, N)>0, R$ being! suitably chosen later. We denote zeros by $a_{n}$ and poles by $b_{m}$. Let

$$
f_{1}(z)=\prod_{n=1}^{N}\left(1-\frac{z}{a_{n}}\right) / \prod_{m=1}^{M}\left(1-\frac{z}{b_{m}}\right),
$$




$$
f_{2}(z)=\prod_{n=1}^{N}\left(1+\frac{z}{\left|a_{n}\right|}\right) / \prod_{m=1}^{M}\left(1-\frac{z}{\left|b_{m}\right|}\right),
$$

and define $f_{3}(z)$ by

$$
f(z)=f_{1}(z) f_{3}(z) \text {. }
$$

Then for $0<r<\frac{1}{2} R$, [3, Lemma A]

$$
\log \left|f_{3}\left(r e^{i \theta}\right)\right|<\frac{14 T(2 R)}{R} r .
$$

Now

(6.3) $T\left(R, f_{2}\right) \leqq N(R, 0)+N(R, \infty)+\sum_{n=1}^{N} \log \left(1+\frac{R}{\left|a_{n}\right|}\right)+\sum_{m=1}^{M} \log \left(1+\frac{R}{\left|b_{m}\right|}\right)$ and

$$
\begin{aligned}
\sum_{n=1}^{N} \log \left(1+\frac{R}{a_{n}}\right) & =\int_{0}^{R} \log \left(1+\frac{r}{t}\right) d n(t, 0) \\
& <n(R, 0) \log 2+\int_{0}^{R} \frac{R}{R+t} \frac{n(t, 0) d t}{t} \\
& <T(2 R, f)+N(R, 0) \quad \text { by }(5.2) \\
& <2 T(2 R, f) .
\end{aligned}
$$

Thus from (6.3) we obtain

$$
T\left(R, f_{2}\right) \leqq 6 T(2 R, f) .
$$

Also, by a result of Edrei [3, formula 8.4], we have for $r \leqq \frac{1}{2} R$

$$
T(r, f) \leqq T\left(r, f_{2}\right)+\frac{14 r}{R} T(2 R, f) .
$$

We now apply Lemma 1 to $f_{2}(z)$, which satisfies the hypotheses, to obtain, for any $r_{1}, r_{2}, 0<r_{1}<r_{2}<R$,

$$
\begin{gathered}
\int_{r_{1}}^{r_{2}}\left(\pi \rho N(r, 0)-\sin \pi \rho \log \left|f_{2}(r)\right|-\pi \rho \cos \pi \rho N(r, \infty)\right) \frac{d r}{r^{1+\rho}} \\
>k \frac{T\left(r_{1}, f_{2}\right)}{r_{1}^{\rho}}-K \frac{T\left(2 r_{2}, f_{2}\right)}{r_{2}^{\rho}},
\end{gathered}
$$

where $k, K$ depend only on $f_{2}$, i.e. on $f$. Thus by our hypothesis (4.1)

$$
\begin{gathered}
0 \geqq \sin \pi \rho \int_{r_{1}}^{r_{2}}\left(\log \left|f_{2}(r)\right|-\log M(r, f)\right) \frac{d r}{r^{1+\rho}} \\
+k \frac{T\left(r_{1}, f_{2}\right)}{r_{1}^{\rho}}-K \frac{T\left(2 r_{2}, f_{2}\right)}{r_{2}^{\rho}} .
\end{gathered}
$$


But from (6.1), i.e.

$$
\log M(r, f) \leqq \log M\left(r, f_{1}\right)+\log M\left(r, f_{3}\right) \leqq \log \left|f_{2}(r)\right|+\log M\left(r, f_{3}\right)
$$

$$
\log \left|f_{2}(r)\right|-\log M(r, f) \geqq-\log M\left(r, f_{3}\right) .
$$

Thus for $r_{2} \leqq \frac{1}{2} R$ we obtain by (6.2),

$$
\begin{aligned}
\sin \pi \rho \int_{r_{1}}^{r_{2}}\left(\log \left|f_{2}(r)\right|-\log M(r, f)\right) \frac{d r}{r^{1+\rho}}>-14 \sin \pi \rho \frac{T(2 R)}{R} \int_{r_{1}}^{r_{2}} \frac{d r}{r^{\rho}} \\
=\frac{-14 \sin \pi \rho}{1-\rho} \frac{T(2 R)}{R}\left\{r_{2}^{1-\rho}-r_{1}^{1-\rho}\right\} \\
\frac{-14 \sin \pi \rho}{1-\rho} \frac{T(2 R)}{R} r_{2}^{1-\rho} .
\end{aligned}
$$

If we now choose $r_{2}=\frac{1}{2} R$ we obtain from (6.6)

$$
0 \geqq k \frac{T\left(r_{1}, f_{2}\right)}{r_{1}^{\rho}}-2^{\rho} K \frac{T\left(R, f_{2}\right)}{R^{\rho}}-2^{\rho-1} \cdot 14 \frac{\sin \pi \rho}{1-\rho} \frac{T(2 R, f)}{R^{\rho}} .
$$

We now use the estimates (6.4) and (6.5) to obtain

$0 \geqq k \frac{T\left(r_{1}, f\right)}{r_{1}^{\rho}}-14\left(\frac{r_{1}}{R}\right)^{1-\rho} \frac{T(2 R, f)}{R^{\rho}}-\left(6.2^{\rho} K+\frac{14.2^{\rho-1} \sin \pi \rho}{1-\rho}\right) \frac{T(2 R, f)}{R^{\rho}}$.

Finally since $r_{1}<\frac{1}{2} R$ we have, for some suitable constant $K_{1}>0$.

$$
0 \geqq k \frac{T\left(r_{1}, f\right)}{r_{1}^{\rho}}-K_{1} \frac{T(2 R, f)}{(2 R)} \text {. }
$$

This holds for all $R$ and all $r_{1}<\frac{1}{2} R$.

7. Theorem 4 now follows from (6.7). Suppose that $r_{1}$ is fixed, then by the definition of $\beta$ there exist arbitrarily large values of $R$ such that for any $\varepsilon>0$

$$
T(2 R, f)<(\beta+\varepsilon)(2 R)^{\rho} \text {. }
$$

Thus for any $\varepsilon>0$

$$
k \frac{T\left(r_{1}, f\right)}{r_{1}^{\rho}} \leqq K_{1}(\beta+\varepsilon) .
$$

The left-hand side of this inequality is a fixed positive number and so if $\beta=0$ this gives a contradiction. Thus

$$
\beta=\lim _{r \rightarrow \infty} \inf \frac{T(r)}{r^{\rho}}>0 .
$$

Also (7.1) holds for any $r_{1}$. Thus if $\beta<\infty$ we obtain 


$$
\alpha=\lim _{r \rightarrow \infty} \sup \frac{T(r)}{r^{\rho}} \leqq \frac{K_{1} \beta}{k}<\infty .
$$

This completes the proof of Theorem 4.

We note that for a given $f(z)$ explicit values of $K_{1}$ and $k$ could be calculated. This, however, sheds no light on the more interesting question of whether or not $\alpha=\beta$ under the hypotheses of Theorem 4 .

If instead of (4.1) we use the assertions (4.3) and (4.4) we obtain instead of (6.7) the assertions

and

$$
\varepsilon \geqq k \frac{T\left(r_{1}, f\right)}{r_{1}^{\rho}}-K_{1} \frac{T(2 R, f)}{(2 R)^{\rho}}, \quad r_{1}>r(\varepsilon)
$$

$$
\frac{K_{2} \log r_{1}}{r_{1}^{\rho}} \geqq k \frac{T\left(r_{1}, f\right)}{r_{1}^{\rho}}-K_{1} \frac{T(2 R, f)}{(2 R)^{\rho}}
$$

respectively for some $K_{2}<\infty$. The conclusions then follow as before since we are assuming that $f(z)$ is transcendental. Thus Theorem 5 is proved.

8. It remains to prove Theorem 2. By Theorem 4 and (1.1) we have $\beta^{\prime}>0$, and if $\beta^{\prime}<\infty$, then

$$
\alpha^{\prime}=\lim _{r \rightarrow \infty} \sup \frac{\log M(r)}{r^{\rho}}<\infty
$$

and we have to show that $\alpha=\beta$. Now $f(z)$ has genus zero by (8.1). Let

$$
\begin{aligned}
& f(z)=\prod_{n=1}^{\infty}\left(1-\frac{z}{a_{n}}\right), \\
& F(z)=\prod_{n=1}^{\infty}\left(1+\frac{z}{\left|a_{n}\right|}\right) .
\end{aligned}
$$

Then, [8], p. 204, we have

$$
\log M(r, f) \leqq \log F(r),
$$

Hence by (2.1)

$$
N(r, 0) \leqq \frac{\sin \pi \rho}{\pi \rho} \log F(r)
$$

Also from (8.1)

$$
\alpha_{1}=\lim _{r \rightarrow \infty} \sup \frac{\log F(r)}{r^{\rho}}<\infty .
$$

Now $F(z)$ satisfies the hypotheses of Lemma 1, and so, with the notation of that lemma 


$$
\int_{r_{1}}^{r_{2}}(\pi \rho N(r, 0)-\sin \pi \rho \log F(r)) \frac{d r}{r^{1+\rho}}=\frac{Q\left(r_{1}\right)}{r_{1}^{\rho}}-\frac{Q\left(r_{2}\right)}{r_{2}^{\rho}}
$$

Now by (5.2),

and so from (8.4)

$$
Q(r)<K T(2 r)<K \log F(2 r)
$$

$$
\alpha^{\prime \prime}=\lim _{r \rightarrow \infty} \sup \frac{Q(r)}{r^{\rho}}<\infty
$$

By (8.3) we obtain

$$
\frac{Q\left(r_{1}\right)}{r_{1}^{\rho}} \leqq \frac{Q\left(r_{2}\right)}{r_{2}^{\rho}}
$$

for all $r_{2}>r_{1}>0$. Now let $r_{2} \rightarrow \infty$ through a suitable sequence of values so that $r_{2}^{-\rho} Q\left(r_{2}\right)$ tends to its lower limit $\beta^{\prime \prime}$ say. We obtain $\alpha^{\prime \prime} \leqq \beta^{\prime \prime}$ and so $Q(r) \sim \alpha^{\prime \prime} r^{\rho}(r \rightarrow \infty)$.

Applying this to (8.5) we see that given any $\varepsilon>0$ there exists an $r(\varepsilon)$ such that for $r_{2}>r_{1}>r(\varepsilon)$,

$$
\varepsilon>\int_{r_{1}}^{r_{2}}(\pi \rho N(r, 0)-\sin \pi \rho \log F(r)) \frac{d r}{r^{1+\rho}}>-\varepsilon .
$$

By our hypothesis (2.1) and (8.2)

$$
-\varepsilon<\int_{r_{1}}^{r_{2}}(\pi \rho N(r, 0)-\sin \pi \rho \log M(r, f)) \frac{d r}{r^{1+\rho}} \leqq 0
$$

and so we obtain by subtraction that

$$
0 \geqq \sin \pi \rho \int_{r_{1}}^{r_{2}}(\log F(r)-\log M(r, f)) \frac{d r}{r^{1+\rho}} \geqq-2 \varepsilon .
$$

Thus the integral

$$
\int_{0}^{\infty}(\log F(r)-\log M(r, f)) \frac{d r}{r^{1+\rho}}
$$

exists and is finite. Now,

$$
\log F(r)=r \int_{0}^{\infty} \frac{n(t, 0) d t}{t(t+r)}=r \int_{0}^{\infty} \frac{N(t, 0) d t}{(t+r)^{2}}
$$

and so we may write (8.3) as

$$
\begin{aligned}
r^{-\rho} N(r, 0) & \leqq \frac{\sin \pi \rho}{\pi \rho} r^{1-\rho} \int_{0}^{\infty} \frac{N(t, 0)}{t^{\rho}} \frac{t^{\rho} d t}{(t+r)^{2}} \\
& =\frac{\sin \pi \rho}{\pi \rho} \int_{0}^{\infty} \frac{N(t, 0)}{t^{\rho}} \frac{(t / r)^{1+\rho}}{((t / r)+1)^{2}} \frac{d t}{t} .
\end{aligned}
$$


We let $r=e^{s}, t=e^{u}$ and let $\psi(s)=r^{-\rho} N(r, 0)$. Thus (8.3) becomes

$$
\psi(s) \leqq \frac{\sin \pi \rho}{\pi \rho} \int_{-\infty}^{\infty} \psi(u) \frac{\left(e^{u-s}\right)^{1+\rho}}{\left(e^{n-s}+1\right)^{2}}
$$

i.e.

$$
\psi(s) \leqq \int_{-\infty}^{\infty} \psi(u) K(u-s) d u
$$

where

$$
K(x)=\frac{\sin \pi \rho}{\pi \rho} e^{x(1+\rho)}\left(e^{x}+1\right)^{-2} .
$$

Convolution inequalities like (8.5) have been studied by Essén [4]. He has the following lemma.

LEMMA 2. Let $\psi(s)$ be bounded and slowly decreasing, i.e.

$$
\liminf _{x \rightarrow \infty} \liminf _{y \rightarrow x \rightarrow 0: y>x}|\psi(y)-\psi(x)| \geqq 0 .
$$

If $K(x) \in \mathscr{L}(-\infty, \infty)$ and satisfies

$$
\begin{gathered}
\int_{-\infty}^{\infty} K(x) d x=1, \\
\int_{-\infty}^{\infty}|x| K(x) d x<\infty, \\
\int_{-\infty}^{\infty} x K(x) d x=m \neq 0,
\end{gathered}
$$

then the inequality (8.5) implies that $\lim _{x \rightarrow \infty} \psi(x)$ exists.

If we apply the lemma to $\psi(s)=r^{-\rho} N(r, 0)$ as above, we obtain

$$
N(r, 0) \sim \operatorname{lr}^{\rho} \quad(r \rightarrow \infty)
$$

for some $l$. We must show that $\psi$ and $K$ satisfy the hypothesis of the lemma. It is easy to verify that

$$
\int_{-\infty}^{\infty} \frac{e^{x(1+\rho)} d x}{\left(e^{x}+1\right)^{2}}=\pi \rho(\operatorname{cosec} \pi \rho)
$$

Differentiating with respect to $\rho$ we obtain $(0<\rho<1)$

$$
\int_{-\infty}^{\infty} \frac{x e^{x(1+\rho)} d x}{\left(e^{x}+1\right)^{2}}=\pi(\sin \pi \rho-\pi \rho \cos \pi \rho) \operatorname{cosec}^{2} \pi \rho \neq 0 .
$$


The other condition on $K$ is also clearly satisfied. It follows from (8.1) that $\psi(s)$ is bounded. Also

$$
\psi(y)-\psi(x)=\frac{N\left(r_{2}\right)}{r_{2}^{\rho}}-\frac{N\left(r_{1}\right)}{r_{1}^{\rho}}>\frac{N\left(r_{1}\right)}{r_{1}^{\rho}}\left[\left(\frac{r_{1}}{r_{2}}\right)^{\rho}-1\right] .
$$

As $r_{2} / r_{1} \rightarrow 1$ and $r \rightarrow \infty$ the above expression tends to zero, and so $\psi$ is slowly decreasing. Thus

$$
N(r, 0) \sim l r^{\rho} \quad(r \rightarrow \infty)
$$

and elementary Tauberian and Abelian arguments enable us to conclude that

$$
\begin{aligned}
n(r, 0) & \sim l \rho r^{\rho} & & (r \rightarrow \infty), \\
\log F(r) & \sim \pi l \rho(\operatorname{cosec} \pi \rho) r^{\rho} & & (r \rightarrow \infty) .
\end{aligned}
$$

But from (8.4) we conclude by a well-known argument $[1, \S 4]$ that

$$
\lim r^{-\rho}(\log F(r)-\log M(r, f))=0
$$

as $r \rightarrow \infty$ outside an open set, $E$ say, of finite logarithmic length. Thus for $r \notin E$

$$
\log M(r, f) \sim \pi l \rho(\operatorname{cosec} \pi \rho) r^{\rho} \quad(r \rightarrow \infty) .
$$

But if $r \in E$ there exists $r_{1} r_{2} \notin E$ with $r_{1}<r<r_{2}$ and such that $\log \left(r_{2} / r_{1}\right) \rightarrow 0$ $\left(r_{1} \rightarrow \infty\right)$. Now $\log M(r, f)$ is a monotonic increasing function of $r$. Thus given $\varepsilon>0$ we have, for $r$ sufficiently large,

$$
\log M(r, f)>\log M\left(r_{1}, f\right) \sim \pi l \rho(\operatorname{cosec} \pi \rho) r_{1}^{\rho}>\pi l \rho(\operatorname{cosec} \pi \rho)(1-\varepsilon) r^{\rho}
$$

and

$$
\log M(r, f)<\log M\left(r_{2}, f\right) \sim \pi l \rho(\operatorname{cosec} \pi \rho) r_{2}^{\rho}<\pi l \rho(\operatorname{cosec} \pi \rho)(1+\varepsilon) r^{\rho} .
$$

Hence

$$
\log M(r, f) \sim \pi l \rho(\operatorname{cosec} \pi \rho) r^{\rho} \quad(r \rightarrow \infty) .
$$

Thus, by the definition of $\beta^{\prime}$,

$$
\begin{array}{rlrl}
\log M(r, f) & \sim \beta^{\prime} r^{\rho} & & (r \rightarrow \infty), \\
N(r, 0) & \sim \frac{\beta^{\prime} \sin \pi \rho}{\pi \rho} r^{\rho} & (r \rightarrow \infty),
\end{array}
$$

which is the required result.

\section{REFERENCES}

1. J. M. Anderson, Growth properties of integral and subharmonic functions, J. Analyse Math. 13 (1964), 355-389.

2. N. A. Bowen, A function-theory proof of Tauberian theorems on integral functions, Quart. J. Math. Oxford Ser. 19 (1948), 90-100. 
3. A. Edrei, The deficiencies of meromorphic functions of finite lower order, Duke Math. J. 31 (1964), 1-21.

4. M. Essén, Note on "A theorem on the minimum modules of entire functions" by Kjellberg, Math. Scand. 12 (1963), 12-14.

5. A. A. Gol'dberg, Growth of an entire function along a half-line, Dokl. Akad. Nauk SSSR 152 (1963), 1049-1050. (Russian)

6. A. A. Gol'dberg and I. V. Ostrovskii, Some theorems on the growth of meromorphic functions, Kharkov Gos. Univ. Uč. Zap. 4 (1961), 27.

7. W. K. Hayman, Meromorphic functions, Oxford Univ. Press, New York, 1964.

8. M. Heins, Entire functions with bounded minimum modulus; subharmonic function analogues, Ann. of Math. 49 (1948), 200-213.

9. B. Kjellberg, On the minimum modulus of entire functions of lower order less than one, Math. Scand. 8 (1960), 189-197. 5-11.

10. — - A theorem on the minimum modulus of entire functions, Math. Scand. 12 (1963),

11. I. V. Ostrovskii, On defects of meromorphic functions with lower order less than one, Dokl. Akad. Nauk SSSR 150 (1963), 32-35. (Russian)

12. R. E. A. C. Paley, A note on integral functions, Proc. Cambridge Philos. Soc. 28 (1932), 262-265.

13. G. Valiron, Sur le minimum du module des fonctions entières d'ordre inferieur d un, Mathematica 11 (1935), 264-269.

14. A. Wahlund, Über einem Zusammenhang zwischen dem Maximalbetrage der ganzen Funktion und seiner unteren Grenze nach dem Jensen'schen Theoreme, Ark. Mat. Astronom. Fys. 21A, No. 23 (1929), 34 pp.

\section{HARVARD UNIVERSITY, CAMBridge, MassachusetTs}

